\title{
Forefoot deformation during the stance phase of normal gait
}

\author{
Saartje Duerinck ${ }^{1,2^{*}}$, Friso Hagman ${ }^{3}$, Ilse Jonkers ${ }^{4}$, Peter Vaes ${ }^{2}$, Peter Van Roy ${ }^{1}$ \\ From 3rd Congress of the International Foot and Ankle Biomechanics Community \\ Sydney, Australia. 11-13 April 2012
}

\section{Background}

During human walking the ankle-foot complex executes seemingly contradictory functions: (1) stabilization of the human body at initial contact, (2) shock absorption during early stance [1-3], (3) Storing elastic energy during midstance and (4) providing a strong lever for push of during final stance [1]. This quadrupled function inevitably demands a transfer from a flexible and compliant foot towards a rigid lever [1]. Despite the viable role of the forefoot in this transfer, knowledge concerning the deformation of the forefoot is limited. The aim of this study is to provide a more detailed description of deformation occurring at the level of the forefoot during the stance phase of normal human walking.

\section{Materials and methods}

Using a seven-camera motion capture system $(250 \mathrm{~Hz})$, a pressure platform $(500 \mathrm{~Hz})$ and a forceplate $(1250 \mathrm{~Hz})$, we measured forefoot deformation through kinematic and pressure related outcome measures in 60 healthy subjects.

\section{Results}

Small but significant changes in intermetatarsal distance are established during stance phase, with the largest change occurring between metatarsal head II/III and V (Table 1). The changes in intermetatarsal distance and metatarsal arch height show slightly different patterns. Both patterns are characterized by a rapid increase in

Table 1 Parameters characterizing the changes in medio-lateral arch height and mutual distances between metatarsal head I, II/III and V and metatarsal base I and V during stance phase and for the different subphases

\begin{tabular}{ccccccc}
\hline & StPh $(\mathbf{m m})$ & HC $(\mathbf{m m})$ & MF $(\mathbf{m m})$ & MS $(\mathbf{m m})$ & IPO $(\mathbf{m m})$ & FPO $(\mathbf{m m})$ \\
\hline Max. MedioLat Height & $1.13 \pm 0.08$ & $0.87 \pm 0.07$ & $0.87 \pm 0.06$ & $1.01 \pm 0.04$ & $1.13 \pm 0.08$ & $1.05 \pm 0.10$ \\
Min. MedioLat Height & $85.95 \pm 8.95$ & $4.39 \pm 2.50$ & $12.34 \pm 3.32$ & $47.25 \pm 12.02$ & $87.39 \pm 7.73$ & $95.88 \pm 1.27$ \\
Max. distance HMTI-HMTV & $1.01 \pm 0.01$ & $0.92 \pm 0.02$ & $0.96 \pm 0.02$ & $1.01 \pm 0.01$ & $1.00 \pm 0.01$ & $0.94 \pm 0.02$ \\
Min. distance HMTI-HMTV & $0.90 \pm 0.02$ & $0.90 \pm 0.02$ & $0.92 \pm 0.02$ & $0.96 \pm 0.02$ & $0.94 \pm 0.02$ & $0.91 \pm 0.02$ \\
Max. distance HMTI-HMTII/III & $1.01 \pm 0.04$ & $0.94 \pm 0.04$ & $0.95 \pm 0.04$ & $1.01 \pm 0.03$ & $1.01 \pm 0.02$ & $0.97 \pm 0.04$ \\
Min. distance HMTI-HMTII/III & $0.91 \pm 0.04$ & $0.92 \pm 0.04$ & $0.93 \pm 0.04$ & $0.95 \pm 0.04$ & $0.97 \pm 0.04$ & $0.93 \pm 0.04$ \\
Max. distance HMTII/III- HMTV & $1.01 \pm 0.04$ & $0.89 \pm 0.05$ & $0.94 \pm 0.05$ & $1.01 \pm 0.04$ & $1.01 \pm 0.04$ & $0.93 \pm 0.04$ \\
Min. distance HMTII/III- HMTV & $0.87 \pm 0.05$ & $0.87 \pm 0.05$ & $0.89 \pm 0.05$ & $0.94 \pm 0.48$ & $0.93 \pm 0.04$ & $0.90 \pm 0.04$ \\
Max. distance BMTI-BMTV & $1.00 \pm 0.01$ & $0.99 \pm 0.01$ & $0.99 \pm 0.01$ & $1.00 \pm 0.01$ & $1.00 \pm 0.01$ & $1.00 \pm 0.01$ \\
Min. distance BMTI-BMTV & $0.97 \pm 0.01$ & $0.97 \pm 0.01$ & $0.99 \pm 0.01$ & $0.99 \pm 0.01$ & $0.98 \pm 0.01$ & $0.97 \pm 0.01$ \\
\hline
\end{tabular}

Legend: $\mathrm{StPh}=$ stance phase, $\mathrm{HC}=$ heel contact, $\mathrm{MF}=$ metatarsal forming, $\mathrm{MS}=$ midstance, $\mathrm{IPO}=$ initial propulsion, $\mathrm{FPO}=$ final propulsion, max. $=$ maximum, min. = minimum, $\mathrm{HMT}=$ head metatarsal, $\mathrm{BMT}$ = base metatarsal

\footnotetext{
* Correspondence: sduerinc@vub.ac.be

${ }^{1}$ Department of Experimental Anatomy, Vrije Universiteit Brussel, Brussels,

1090, Belgium

Full list of author information is available at the end of the article
}

\section{() Biomed Central}

C 2012 Saartje et al; licensee BioMed Central Ltd. This is an Open Access article distributed under the terms of the Creative Commons Attribution License (http://creativecommons.org/licenses/by/2.0), which permits unrestricted use, distribution, and reproduction in any medium, provided the original work is properly cited. 


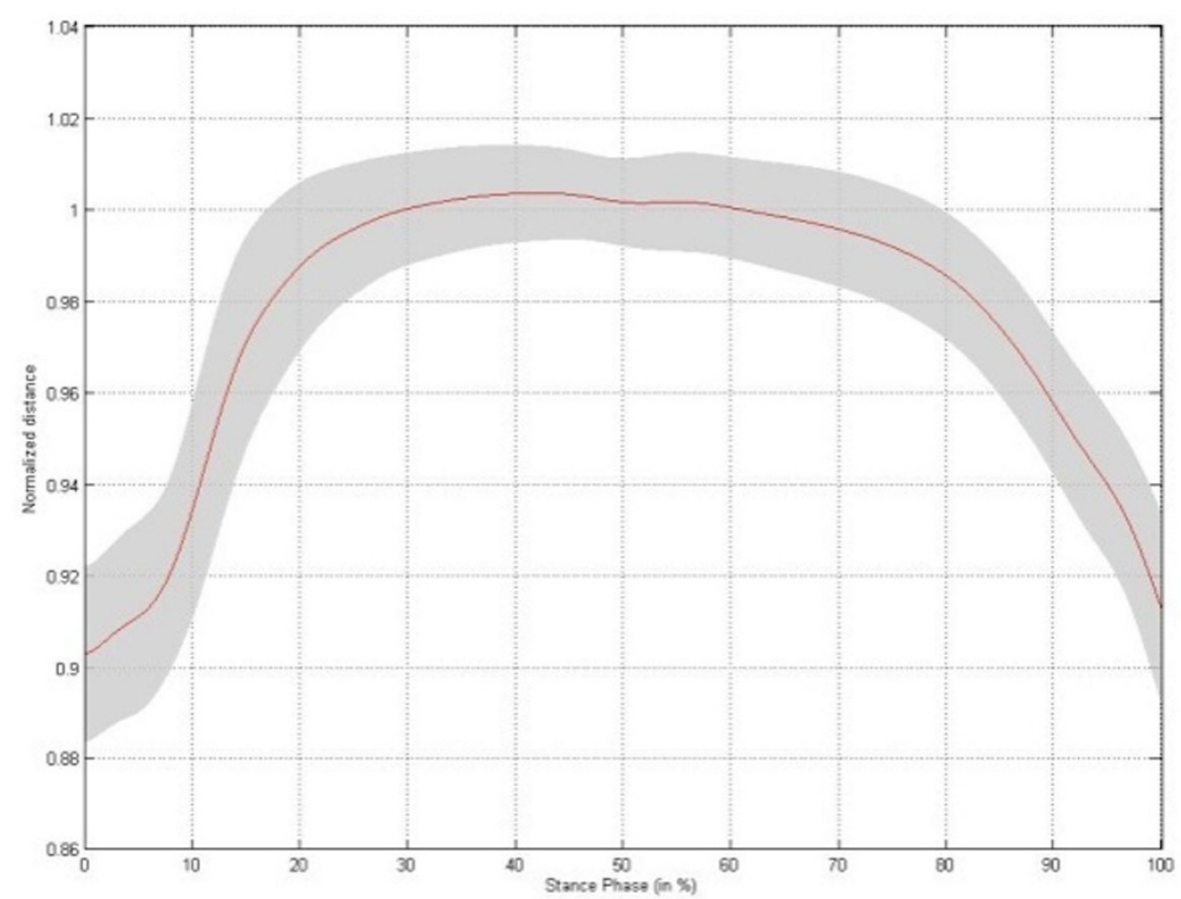

Figure 1 Changes in distance between metatarsal head I - V, I - II/II and II/III - V and in metatarsal arch height: Changes in distance between metatarsal head I and metatarsal head $V$ throughout stance phase for the left foot,

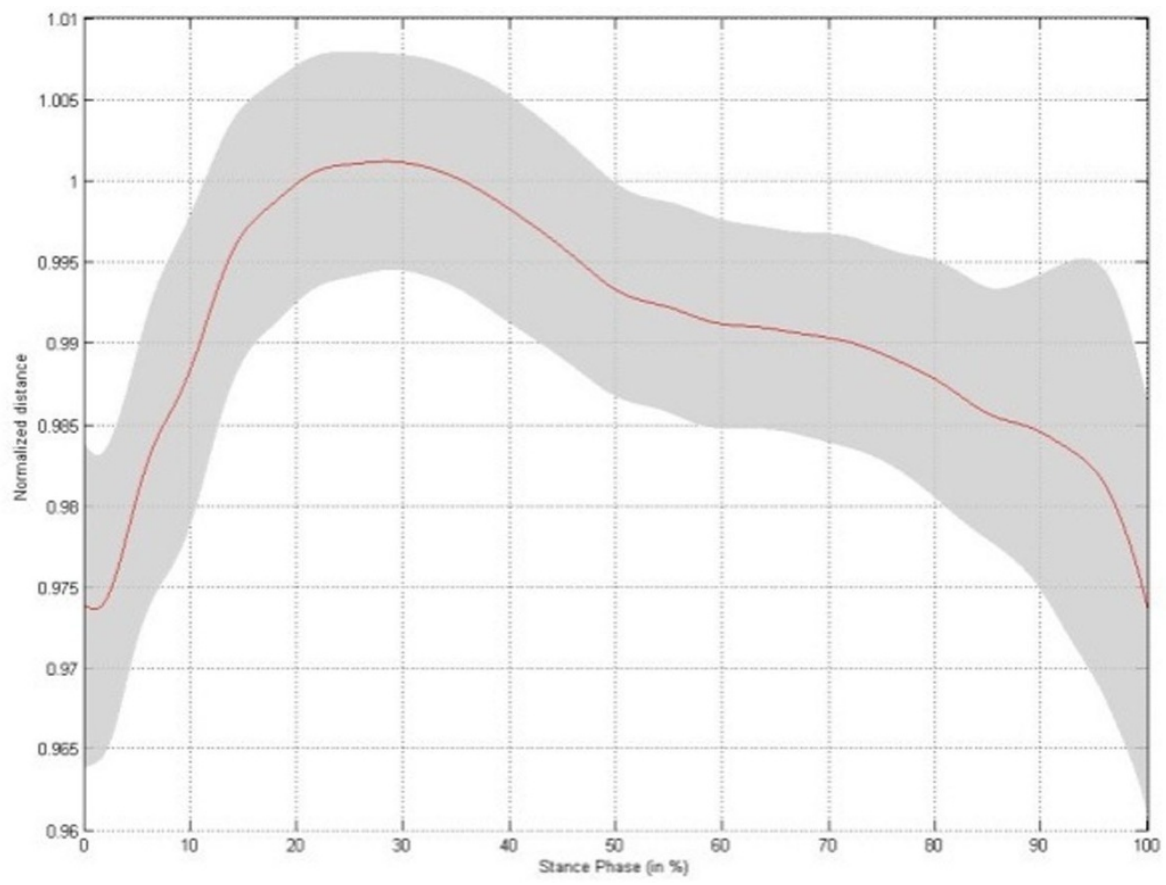

Figure 2 Changes in distance between the base of metatarsal I and the base of metatarsal $V$ throughout stance phase for the left foot, 


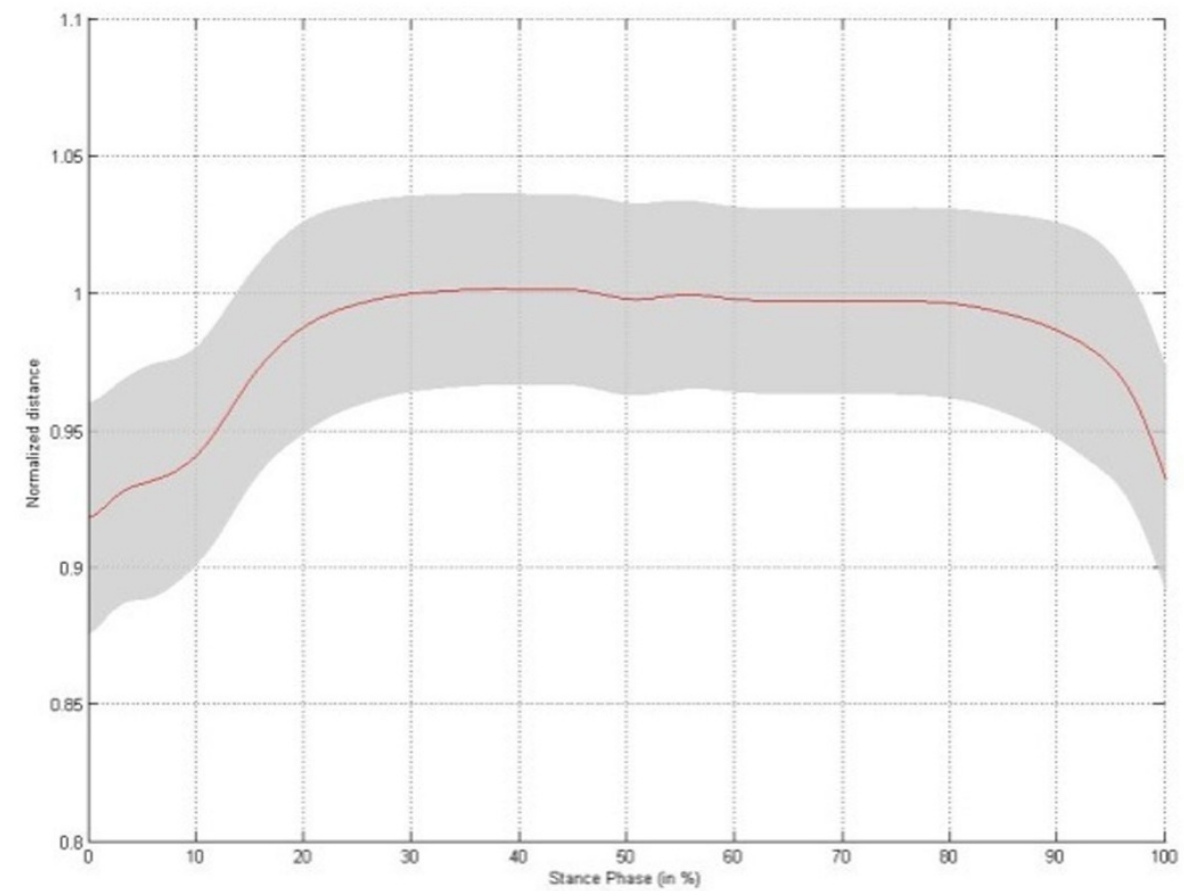

Figure 3 Changes in distance between metatarsal head I and metatarsal head II/III throughout stance phase for the left foot,

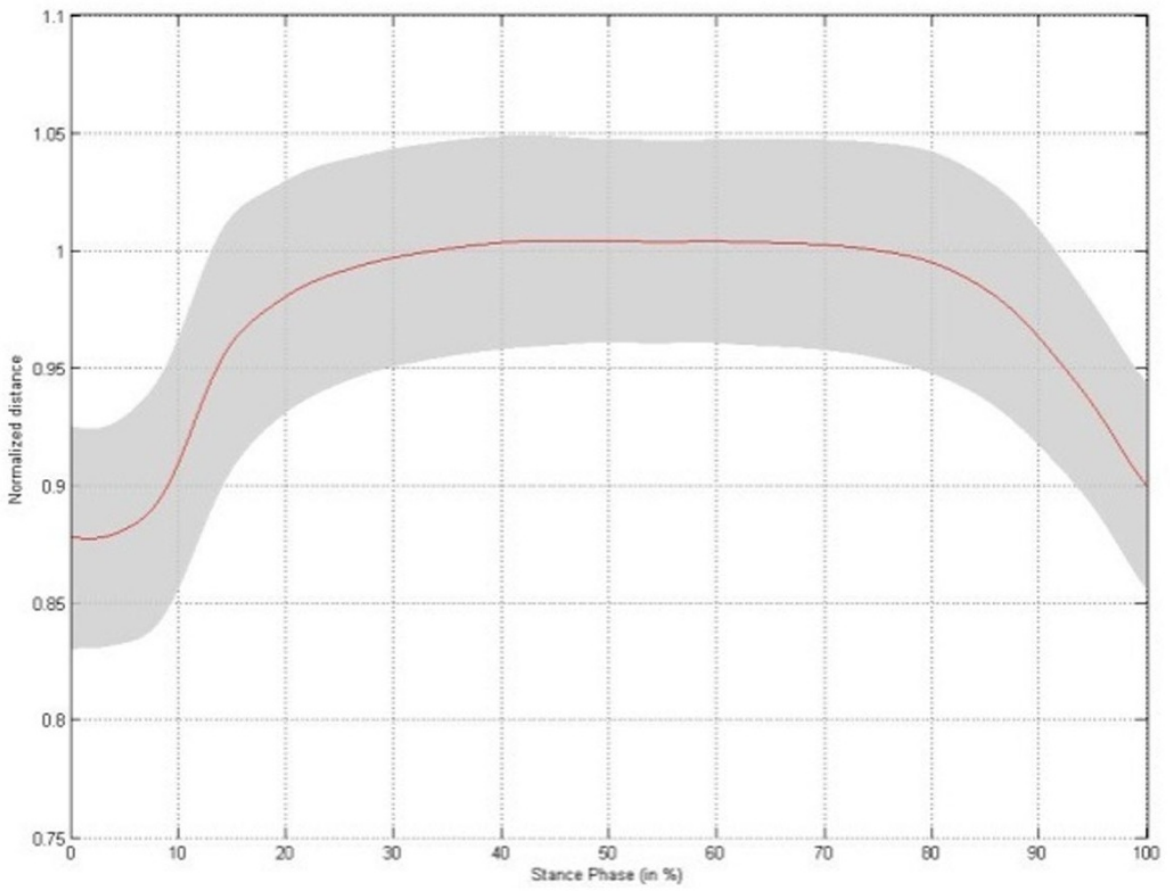

Figure 4 Changes in distance between metatarsal head II/III and metatarsal head V throughout stance phase for the left foot 


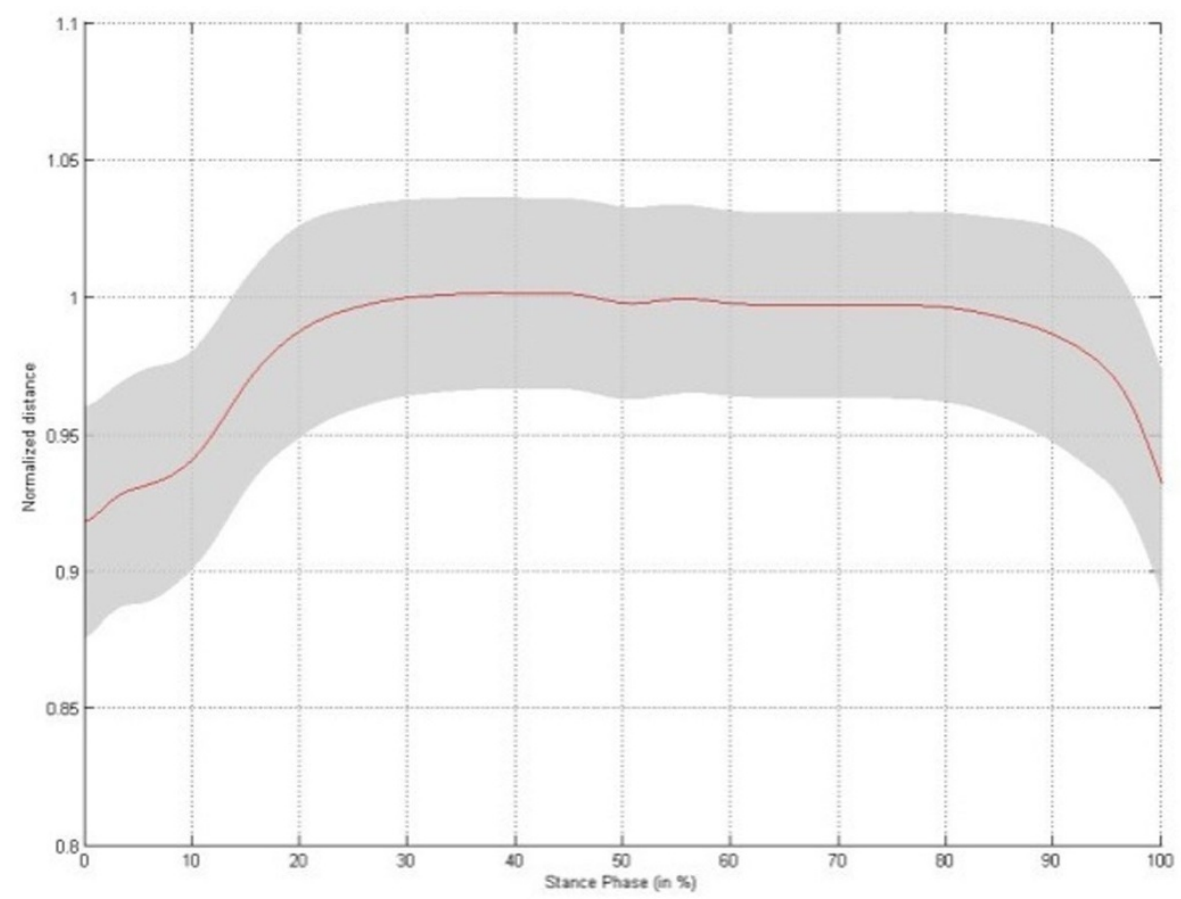

Figure 5 Changes in medio-lateral arch height throughout stance phase for the left foot

distance during initial stance, reaching a stable platform throughout midstance. At the end of stance phase the intermetatarsal distances rapidly decrease to baseline, whereas the metatarsal arch height increases till a maximum at heel off (Figure 1-5).

High correlation values $(>0.7$ or $<-0.7)$ are found between temporal pressure and temporal kinematic parameters.

\section{Conclusion}

Through stance the forefoot deforms according to a specific pattern, which is predominantly determined through forefoot-ground interaction. In addition, the changes in forefoot kinematics in combination with temporal contact data argue the existence of a mediolateral metatarsal arch and suggest the existence of an inverse arch during metatarsal forming and final propulsion phase.

\section{Acknowledgement}

The preparation of this abstract was funded by the Vrije Universiteit Brussel (i.e., GOA 59)

\section{Author details}

'Department of Experimental Anatomy, Vrije Universiteit Brussel, Brussels, 1090, Belgium. ${ }^{2}$ Department of Physical Therapy, Vrije Universiteit Brussel, Brussels, 1090, Belgium. ${ }^{3}$ Department of Human Biomechanics \& Biometrics, Vrije Universiteit Brussel, Brussels, 1090, Belgium. ${ }^{4}$ Department of Biomedical Kinesiology, Katholieke Universiteit Leuven Belgium, Leuven, 3000, Belgium.

Published: 10 April 2012

\section{References}

1. Jenkyn TR, Anas K, Nichol A: Foot segment kinematics during normal walking using a multisegment model of the foot and ankle complex. J Biomech Eng 2009, 131:034504.

2. Winter DA: Energy generation and absorption at the ankle and knee during fast, natural, and slow cadences. Clin Orthop Relat Res 1983, 131:147-154.

3. Ren $L H D$, Ren $L Q$, Nester C, Tian LM: a phase-dependent hypothesis for locomotor functions of human foot complex. J Bionic Eng 2008, 5:175-180

doi:10.1186/1757-1146-5-S1-P12

Cite this article as: Duerinck et al:: Forefoot deformation during the stance phase of normal gait. Journal of Foot and Ankle Research 20125 (Suppl 1):P12.

\section{Submit your next manuscript to BioMed Central and take full advantage of:}

- Convenient online submission

- Thorough peer review

- No space constraints or color figure charges

- Immediate publication on acceptance

- Inclusion in PubMed, CAS, Scopus and Google Scholar

- Research which is freely available for redistribution 